\title{
Kleine Relativitätstheorie
}

\section{Bernhard Gurtner}

Korrespondenz:

Dr. med. Bernhard Gurtner

Eggstrasse 76

CH-8620 Wetzikon

gurtner.bernhard@bluewin.ch
Ein Stein fiel uns vom Herzen, als wir beim ersten propädeutischen Examen die halbverdauten Wissensbrocken wieder herauswürgen konnten, die uns in den Fächern Chemie, Physik, Botanik und Zoologie während nur zwei Semestern eingetrichtert worden waren. Wenn ich mich richtig erinnere, waren wir durchaus unkritische Konsumenten des vorgesetzten Stoffes.

Wir hatten weder Zeit noch Lust und schon gar nicht die Kompetenz, den Wahrheitsgehalt zu überprüfen. Richtig war, was bei der Prüfung als Antwort akzeptiert wurde. Das Postulat von Karl Popper, demzufolge jede Erfahrungswissenschaft durch neue Erkenntnisse zu «falsifizieren» sei, war uns noch nicht bekannt, und erst allmählich lernten wir, dass auch medizinische Lehrsätze ihre Halbwertszeiten und Verfalldaten haben.

Zunächst aber glaubten wir (fast) alles, was uns gelehrt wurde. Der Anatomieatlas war verlässlich wie eine Wanderkarte der Landestopographie, und falls wir im Seziersaal auf eine atypische Gefässabzweigung stiessen, war diese Anomalie von den Fachleuten bereits beschrieben und benannt worden. Auch die Physiologie und die biochemischen Vorgänge erschienen uns so kompliziert und wunderbar, dass wir keine Veranlassung hatten, die HendersonHasselbalchsche Gleichung oder andere molekulare Stoffwechseltheorien zu bezweifeln. Wir waren als Medizinstudenten sehr gläubige Jünger der Wissenschaft.

Dieses unbedingte Vertrauen übertrug sich auch auf die klinischen Lehrer, doch lernten wir spätestens in den Auslandsemestern, dass sich die Ansichten in Wien oder Paris nicht ganz mit den Aussagen deckten, die wir von den Schweizer Professoren gehört hatten. Es gab geringe Abweichungen und diametrale Gegenmeinungen, die uns verwirrten, aber auch zum Mitdenken anregten. Und so schufen wir uns, ohne es zu merken, eine kleine Relativitätstheorie, die im späteren Berufsleben davor schützte, dogmatischen Behauptungen und Forderungen medizinischer Päpste blind zu folgen.

Rückblickend ist es amüsant zu sehen, welche angeblich unumstösslichen Grundsätze und
Weisungen im Laufe der Jahre verschwunden sind. Manches, was dick umrahmt oder doppelt unterstrichen in den Lehrbüchern oder Kollegheften zu lesen war, erwies sich später als unnötig oder gar als grundfalsch. Herzinfarktpatienten mussten nicht mehr während sechs Wochen im Bett dahinserbeln, sondern wurden früh mobilisiert. Nitroglyzerin in der Akutphase zu verabreichen, galt als Kunstfehler, auch Betablocker schienen im Gegensatz zu heutigen Auffassungen kontraindiziert, dafür wurden Antiarrhythmika bis zum buchstäblichen «Gehtnichtmehr» des Herzens eingesetzt, um böse Extrasystolen zu vertreiben. Die Gastroenterologen predigten schlackenfreie Kost zur Schonung der Kolondivertikel und empfahlen wenige Jahre später faserreiche Ernährung bei der gleichen Indikation. Rheumatologen und Dermatologen lernten, dass kortisonhaltige Präparate auch «in der Hand des Geübten» ihre Gefahren aufweisen und neuerdings mussten die hormonsubstituierenden Gynäkologen studienhalber auf liebgewordene Gewohnheiten verzichten.

Heute gelten EBM und juristisch verpflichtende Guidelines, welche digital gespeichert die voluminösen Blaubücher abgelöst haben, in denen die Ansichten des vorvorletzten Oberarztes auf verblichenen Kopien eingeklemmt waren. Doch sowenig Leitplanken davon dispensieren, selbst zu lenken, so ersparen uns Leitlinien nicht, selbst zu denken. Sie sind kein Massanzug für jeden Einzelfall und kein guter Doktor verpasst nur genormte Konfektionsware. Fingerspitzengefühl ist besser als Faustregeln.

Es lohnt sich, bei jeder Guideline nachzuforschen, von wem sie verfasst worden ist. Wir müssen es wagen, relativierende Fragen $\mathrm{zu}$ stellen und individuell begründbare Modifikationen vorzunehmen, wenn Fachspezialisten, Industrievertreter oder Kostenträger ihre Sonderinteressen in die Richtlinien miteingebracht haben. Und vertrauen wir doch mehr der eigenen Erfahrung, sofern sie sich nicht nur auf die letzten zwei Patienten bezieht, an die wir uns zu erinnern vermögen. 